\title{
Artère labiale de calibre persistant
}

\author{
Lydia Vazquez, Jean-Pierre Carrel, Jacky Samson \\ Division de Stomatologie et Chirurgie orale, Rue Barthélémy-Menn 19, 1205 Genève, Suisse \\ jacky.samson@unige.ch
}

Mots clés : artère de calibre persistant / artère labiale / malformations vasculaires / écho-Doppler couleur

Key words: caliber-persistent artery / labial artery / vascular malformations / color Doppler ultrasound
Résumé - L'artère labiale de calibre persistant semble être relativement fréquente mais rarement décrite, la plupart des cas seraient asymptomatiques. L'écho-Doppler couleur permet de confirmer le diagnostic sans avoir recours à un acte invasif.

Abstract - Caliber-persistent labial artery. The caliber-persitent labial artery appears very common but it is rarely reported; most of the cases are asymptomatic. The color Doppler ultrasound confirm the diagnosis and avoid invasive intervention.

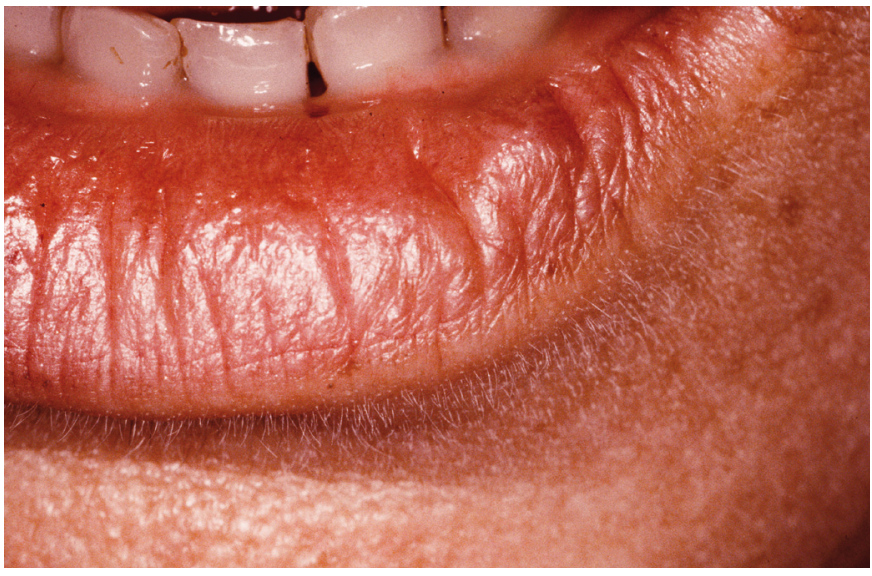

Fig. 1. Petite tumeur labiale inférieure due à une artère de calibre persistant (aspect clinique initial).

Fig. 1. Small inferior labial tumor disclosing to a caliber-persistent artery (initial clinical view).

Une patiente de 52 ans, radiologue spécialiste en échographie, estvenueconsulter, en octobre 2000, pour une tumeur sur la demi-muqueuse labiale inférieure, hémisphérique, de $3 \mathrm{~mm}$ de diamètre, située à $2 \mathrm{~cm}$ de la commissure gauche. Cette tumeur présente depuis 2 ans, recouverte d'une muqueuse normale, n'avait pas changé d'aspect ni de taille (Fig. 1) ; elle était indolore et la patiente percevait parfois des pulsations en appliquant un doigt sur la lésion. La mise en tension de la lèvre entrainait sa disparition complète. Ce tableau clinique faisait suspecter une artère labiale de calibre persistant. En évoquant ce diagnostic, la patiente a proposé de réaliser ellemême un examen écho-Doppler couleur. Cet examen a confirmé le diagnostic en montrant que la tumeur labiale était due à une artère perpendiculaire à la surface muqueuse, de $9 \mathrm{~mm}$ de long et d'un diamètre constant de 1,9 $\mathrm{mm}$. Le diagnostic était confirmé. Devant l'absence de symptomatologie, on a opté pour l'abstention thérapeutique.

Cette patiente a été revue 8 ans plus tard : elle est venue consulter pour une tumeur sessile, médiojugale gauche, de $7 \mathrm{~mm}$ de diamètre. La lésion labiale inférieure était toujours présente et elle n'avait présenté aucune évolution depuis la consultation initiale (Figure de la $1^{\text {re }}$ page de couverture). L'examen histopathologique de la lésion jugale a confirmé qu'il s'agissait bien d'un nodule d'hyperplasie fibro-épithéliale.

\section{Commentaires}

L'artère labiale de calibre persistant a été décrite initialement en 1973 par Howell et Freedman sous le terme de « proeminent inferior labial artery ». C'est Miko et al. qui en 1980 ont proposé le terme « calibre-persistent labial artery » adopté par tous les auteurs anglophones [3]. Certains considèrent que cette anomalie artérielle a été initialement décrite dans la muqueuse gastrique par Gaillard en 1886 [2], mais cet auteur a évoqué plutôt des anévrysmes miliaires qu'on peut difficilement comparer à la lésion unique touchant la lèvre, d'autres qu'elle l'a été par Voth en 1962 [3] qui, d'emblée, proposa le terme de " caliber-persistent artery » pour cette artère anormale qui traversait la couche musculaire et pénétrait dans la sousmuqueuse gastrique, sans division ni réduction de diamètre (d'où la notion de calibre persistant). L'artère de calibre persistant se traduit par une lésion labiale unique (exceptionnellement 
il peut y avoir deux lésions [1]), intéressant plus souvent la demi-muqueuse que le versant muqueux, et plus souvent la lèvre inférieure que la lèvre supérieure. Cette lésion est constituée par une papule arrondie (quelquefois ovale ou linéaire), de quelques $\mathrm{mm}$ de diamètre (de longueur), recouverte par une muqueuse ou une demi-muqueuse d'aspect normal, parfois bleuâtre. Elle peut être pulsatile, à la vue ou à la palpation, spontanément ou après un effort physique. Quelquefois, elle se manifeste par une ulcération chronique. Elle touche le plus souvent des sujets entre 50 et 60 ans; le plus jeune cas publié concernait une jeune femme de 20 ans [1]. Bien que rarement rapportée dans la littérature, sa fréquence serait relativement élevée, de l'ordre de 1 à $2 \%$ (Howell et Freeman in [2], Lovas et al. in [4]).

Histologiquement, on observe une artère avec un diamètre important dont la paroi est remaniée : épaississement ou quelquefois amincissement de la paroi, épaississement de l'intima, fragmentation de la limitante élastique interne, épaississement du muscle lisse, présence de plaques d'athérosclérose avec parfois une occlusion partielle et des petites calcifications [3,4].

Dans le diagnostic différentiel, on peut évoquer une malformation ou une tumeur vasculaire (ectasie vasculaire, anévrysme, hémangiome, hyperplasie endothéliale papillaire intravasculaire), un pseudo-kyste salivaire rétentionnel lorsque la lésion est de couleur bleuâtre, un carcinome épidermoïde lorsqu'il existe une ulcération chronique. En réalité, le diagnostic est rapidement confirmé si on réalise un écho-Doppler couleur : il met en évidence l'artère de calibre persistant et permet de préciser ses dimensions (diamètre et longueur). Il n'est pas nécessaire de recourir à la biopsie pour confirmer le diagnostic $(1,4,5)$. Après la confirmation du diagnostic, on propose l'abstention thérapeutique excepté lorsqu'il existe une ulcération chronique.

L'étiopathogénie de l'artère labiale de calibre persistant reste mal comprise. Plusieurs hypothèses ont été formulées : malformation congénitale, modification de la paroi artérielle avec l'âge ou certains facteurs exogènes (effets induits par les cigarettes, la pipe, l'exposition aux ultraviolets et surtout par les traumatismes répétés) [3]. L'apparition d'une ulcération serait favorisée par l'amincissement de la muqueuse, d'éventuels traumatismes dentaires, la présence de calcifications, et sans doute principalement par la pression systolique qui provoquerait une ischémie de la muqueuse. Les ulcérations seraient plus fréquemment associées aux artères de calibre persistant superficielles et de gros diamètre [2].

Dans le cas rapporté, il existait un tic de succion-aspiration nocturne (hypertrophie massétérine et empreinte des dents sur les bords de la langue) qui a entrainé ultérieurement l'apparition d'un nodule d'hyperplasie fibro-épithéliale. C'est le seul facteur favorisant qui a été retrouvé pour expliquer le développement de cette artère de calibre persistant.

\section{Conflits d'intérêt : aucun}

\section{Références}

1. Kocyigit P, Kocyigit D, Akay BN, Ustuner E, Kisnisci R. Calibre persistent labial artery: clinical features and noninvasive radiological diagnosis. Clin Exp Dermatol 2006;31:528-30.

2. Piccionne MJ, Manganaro AM, Almony JS. Caliber-persistent labial artery: diagnosis and treatment. Case report. J Oral Maxillofac Surg 2010;68:1987-9.

3. Rosdy NMMNM, Firth NA, Rich AM. Calibre-persistent labial artery: often misdiagnosed as a mucocoele. Int J Oral Maxillofac Surg 2010;39:1230-9. available online at http://www.sciencedirect.com

4. Vazquez L, Lombardi T, Guinand-Mkinsi H, Samson J. Ultrasonography: a noninvasive tool to diagnose a caliberpersistent labial artey, an enlarged artery of the lip. J Ultrasound Med 2005;24:1295-301.

5. Wortsman X, Calderon P, Arellano J, Orellana Y. Highresolution color Deppler ultrasound of a caliber-persistent artery of the lip, a simulator variant of dermatologic disease: case report and sonographic findings. Int $\mathrm{J}$ Dermatol 2009;48:830-3. 